\title{
Control of a single Degree of Freedom Noise Rejecting - Variable Impedance Actuator
}

\author{
F. Nori* ${ }^{*}$ B. Berret ${ }^{*}$ L. Fiorio* A. Parmiggiani* G. Sandini* \\ * Department of Robotics, Brain and Cognitive Sciences \\ Italian Institute of Technology \\ Via Morego 30, Genova, ITALY \\ \{francesco.nori,bastien.berret,luca.fiorio,alberto.parmiggiani,giulio.sandini\}@iit.it
}

\begin{abstract}
:
Recently, a number of variable impedance actuator designs have been proposed under different motivations (safe human-robot interaction, mechanical robustness and energy storing to cite a few). In a recent paper (Berret et al. (2011)) we observed that none of the available designs seem to reproduce an important characteristic of human muscles, i.e. the ability to open-loop reject disturbances by means of muscle co-activation. Starting form this observation, we recently designed a novel single-joint actuator (nr-VIA) based on the use of non-linear springs in agonistantagonist configuration. In this paper we discuss some control related characteristics of the proposed design. The theoretical analysis is conducted without specifying the potential energy of the springs. We first design a control law capable of monotonically increasing the joint-stiffness (i.e. disturbance rejection) without changing the joint equilibrium configuration; this result is obtained with minimal requirements on the potential energy of the springs. The same control law is then proven (under more restrictive conditions) to monotonically decrease the sensitivity of the joint equilibrium with respect to the actuation variables, a desirable property when trying to achieve a finer control over joint positioning.
\end{abstract}

Keywords: Actuators, passive compensation, disturbance rejection, springs, sensitivity functions, variable impedance actuators, actuators in agonist-antagonist configuration.

\section{INTRODUCTION}

One of the most important features of a system capable of working in uncertain and unstructured environments is active compliance, or the ability to change the body forcedisplacement characteristic. Today's robots are excellent machines, but are still not able to interact with their surrounding environment, especially when this environment is unstructured. Recent human studies have shown the fundamental role played by muscle co-activation during manipulation tasks (Burdet et al. (2001)) in dealing with unpredictable events. In particular the possibility to vary the stiffness of shoulder, elbow and wrist allow humans to interact easily with fast changing environments and reject unpredictable noise disturbances.

Inspired by these findings, in the last decade roboticists have started designing actuators capable of actively varying their intrinsic compliance, with the intent of reproducing in modern robots the same control capability that humans have, i.e. the ability to change the body intrinsic compliance. These actuators have been sometimes named variable stiffness actuators (VSA, Franklin et al. (2007)) or variable impedance actuators (VIA) or actuator with adjustable stiffness (AwAS, Jafari et al. (2010)), just to cite a few. All these designs, were inspired by three main motivations: energy storing capability, safe human-robot interaction and mechanical robustness. Inspired by these ideas, we recently proposed (Berret et al. (2011)) a differ- ent point of view, suggesting that compliance regulation might also represent a way to deal with unpredictable disturbances in absence of explicit feedback loops. Our main motivation comes from the observation that humans and animals, although slow in closing position feedback loops (typical delays are in the order of one hundred milliseconds), are able to interact easily and reliably with highly unstable force fields Burdet et al. (2001). In order to replicate a similar capability in robots, we recently designed a novel actuator based on an agonist-antagonist configuration. In this paper we present some control properties of this actuator, named Noise Rejecting - Variable Impedance Actuator (nr-VIA) to underline its intrinsic property of dealing with unpredictable events. The specific design choices adopted in the nr-VIA, will be kept out of the present paper because they are currently under a patenting procedure. The main goal of the work will be to present some characteristics of the the proposed actuator which turn out to be very useful in controlling the actuator itself.

The paper is organized as follows. After a preliminary discussion on the analogies between the proposed actuator and biological muscles, Section 3 presents the dynamical model of the actuator based on an under actuated joint attached to an agonist and an antagonist motor group. Section 4, deals with the problem of computing the agonistantagonist coordination (i.e. muscle co-activation) in order 

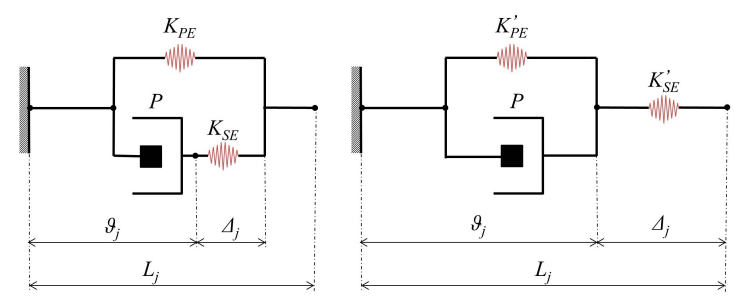

Fig. 1. Hill muscle model (right hand side) and an equivalent model (left hand side).

not to change the joint position. Section 5, shows that the very same agonist-antagonist co-activation monotonically increases the joint stiffness, i.e. its intrinsic torquedisplacement characteristic. Finally, Section 6 shows that muscle co-activation can also be used to decrease the sensitivity of the joint equilibrium with respect to changes in the agonist (or antagonist) activation.

\section{MODELING THE ACTUATOR}

The main properties of the biological muscles have been reported by Hill and Gasser (1924) using the model represented in the left part of Fig.1, used to model the tension dynamics of various isolated frog muscles. It can be proven (see McMahon (1984) pag.23) that its mechanical model is equivalent to the one shown in the right part of Fig. 1 and therefore the overall muscle force can be written as:

$$
F=F_{S E}\left(K_{S E}, \Delta_{j}\right)=F_{P E}\left(K_{P E}, \vartheta_{j}\right)+P\left(L_{j}, f(t)\right),
$$

where $K_{S E}$ is the series nonlinear elastic element, $K_{P E}$ is the parallel nonlinear elastic element which in series with $K_{S E}$ account for the passive tension properties of the muscle and $P$ is the active force generated by the contractile element depending on the muscle history activation $f(t)$ and the overall length $L_{j}$.

During the actuator design we concentrate on two main characteristics of the mechanical muscle model: the possibility of finding a close path that connects the frame to the actuator endpoint and the capability of varying the stiffness independently from length. In reflexive muscle this regulation, as suggested by Feldman (1966), is done controlling the system as a nonlinear spring with adjustable resting length. Thus our goal has been to design a mechanism composed by a nonlinear spring in series with a structure composed by a contractile element in parallel with a second nonlinear spring having the possibility of varying the resting length of the series element. As shown in Fig. 2 we came out with two different solutions both composed by two springs and a cable winder. In particular the "piping winder" works transferring cable from one spring to the other, while the "counter winder" stretches or releases both springs at the same time. To compare the two working principles, it can be useful to consider the effect of a clockwise rotation of the motor winder $\vartheta$ (keeping the joint angular position $q$ constant): for the "counter winder" both springs are stretched increasing their stiffness, while the "piping winder" stretches only the spring $K_{P E}$ transferring cable to the opposite one.

Let's compute the dynamics of the two different designs using the Lagrangian formalism. Variables have the meaning

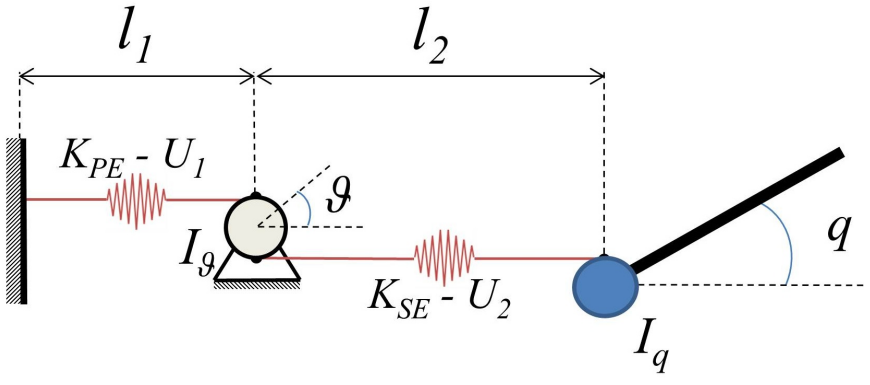

(a) Counter winder

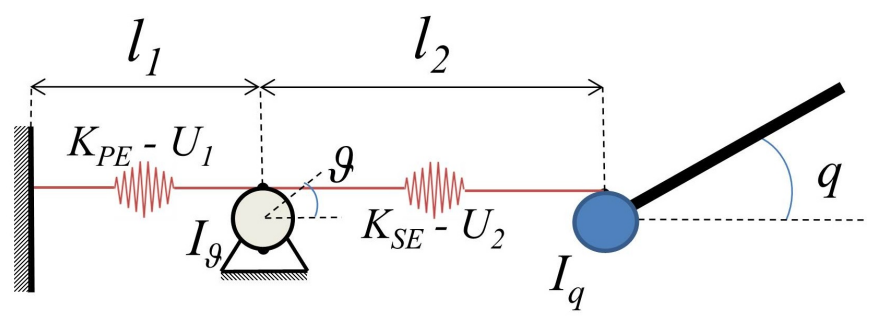

(b) Piping winder

Fig. 2. Different cable winders

shown in Fig. 2, $\vartheta$ is the position of the contractile element, $\tau_{\vartheta}$ is the associated torque, $q$ is the joint position, $\tau_{q}$ the associated torque, $I_{\vartheta}$ the inertia of the contractile element, $R_{\vartheta}$ the radius of the pulley at the contractile element, $R_{q}$ the radius of the joint pulley, $I_{q}$ the joint inertia, $U_{1}$ and $U_{2}$ the potential energies of the springs and $l_{1}$ and $l_{2}$ their length. To simplify the notation let's define a flag variable $\lambda$ to take into account the different configuration of the springs:

Piping winder: $\lambda=+1$, Counter winder: $\lambda=-1$.

The kinematic energy is given by:

$$
K=\frac{1}{2} I_{\vartheta} \dot{\vartheta}^{2}+\frac{1}{2} I_{q} \dot{q}^{2}
$$

while the potential terms:

$$
\begin{aligned}
& U=U_{1}+U_{2} \\
& U_{1}=U_{1}\left(l_{1}\right)=U_{1}\left(-R_{\vartheta} \vartheta\right) \\
& U_{2}=U_{2}\left(l_{2}\right)=U_{2}\left(\lambda R_{\vartheta} \vartheta-R_{q} q\right) .
\end{aligned}
$$

By applying the Lagrangian formalism is possible to obtain the following system dynamics:

$$
\left\{\begin{array}{l}
I_{\vartheta} \ddot{\vartheta}-R_{\vartheta} \frac{\partial U_{1}}{\partial l_{1}}\left(-R_{\vartheta} \vartheta\right)+\lambda R_{\vartheta} \frac{\partial U_{2}}{\partial l_{2}}\left(\lambda R_{\vartheta} \vartheta-R_{q} q\right)=\tau_{\vartheta} \\
I_{q} \ddot{q}-R_{q} \frac{\partial U_{2}}{\partial l_{2}}\left(\lambda R_{\vartheta} \vartheta-R_{q} q\right)=\tau_{q}
\end{array}\right.
$$

In the case of linear springs is possible to use the following simplification:

$$
\frac{\partial U}{\partial l}=K l,
$$

Furthermore assuming $R_{\vartheta}=R_{q}=1$ the system (1) becomes:

$$
\left\{\begin{array}{l}
I_{\vartheta} \ddot{\vartheta}+K_{1} \vartheta+K_{2}(\vartheta-\lambda q)=\tau_{\vartheta} \\
I_{q} \ddot{q}+K_{2}(q-\lambda \vartheta)=\tau_{q}
\end{array}\right.
$$


Considering a constant load $\tau_{\vartheta}=\bar{\tau}_{\vartheta}, \tau_{q}=\bar{\tau}_{q}$ and the system at equilibrium $\ddot{\vartheta}=\ddot{q}=0$ (note that since $\lambda= \pm 1$, we have $\left.\lambda=\lambda^{-1}\right)$ :

$$
\begin{gathered}
\left\{\begin{array}{l}
K_{1} \vartheta+K_{2}(\vartheta-\lambda q)=\bar{\tau}_{\vartheta} \\
K_{2}(q-\lambda \vartheta)=\bar{\tau}_{q}
\end{array}\right. \\
\rightarrow K_{1} \vartheta-\lambda \bar{\tau}_{q}=\bar{\tau}_{\vartheta} \rightarrow \vartheta=\frac{\bar{\tau}_{\vartheta}+\lambda \bar{\tau}_{q}}{K_{1}}
\end{gathered}
$$

and from second equation:

$$
q=\underbrace{\frac{K_{1}+K_{2}}{K_{1} K_{2}}}_{\text {Series } K_{1} \text { and } K_{2}} \cdot \bar{\tau}_{q}+\lambda \frac{\bar{\tau}_{\vartheta}}{K_{1}}
$$

Remarkably, the equivalent stiffness felt at the joint side is the series of $K_{1}$ and $K_{2}$ regardless of the chosen configuration. Between the two solutions the counter winder has been chosen because of the possibility of stretching both springs at the same time avoiding tendon collapse problem during joint $q$ movement and increasing the overall path stiffness. In the next section we connect a second muscle to the joint in order to have an agonist-antagonist configuration.

\section{EQUILIBRIUM CONFIGURATION}

In this section, we characterize the equilibrium configuration of the agonist system represented in Fig. 3. The variable to be controlled is represented by $q$, the joint position, for which no direct control is available. Actuation is instead available on $\vartheta$ and $\vartheta^{a}$ to be thought as internal variables. The system dynamics are:

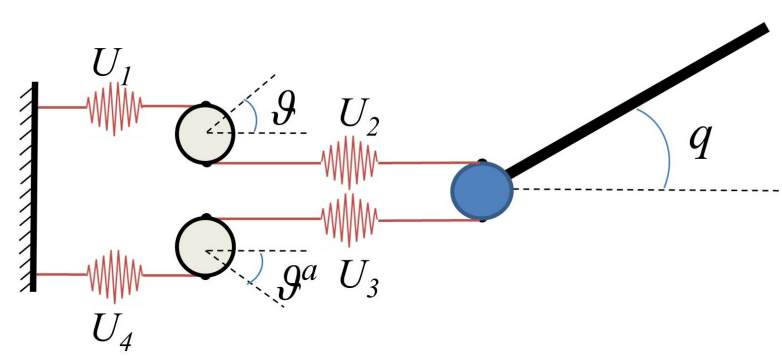

Fig. 3. Complete system with antagonist muscle

$$
\left\{\begin{array}{l}
I_{\vartheta} \ddot{\vartheta}-R_{\vartheta} \frac{\partial U_{1}}{\partial l_{1}}\left(-R_{\vartheta} \vartheta\right)-R_{\vartheta} \frac{\partial U_{2}}{\partial l_{2}}\left(-R_{\vartheta} \vartheta-R_{q} q\right)=\tau_{\vartheta} \\
I_{q} \ddot{q}-R_{q} \frac{\partial U_{2}}{\partial l_{2}}\left(-R_{\vartheta} \vartheta-R_{q} q\right)+R_{q} \frac{\partial U_{3}}{\partial l_{3}}\left(R_{q} q+R_{\vartheta} \vartheta^{a}\right)=\tau_{q} \\
I_{\vartheta} \ddot{\vartheta}^{a}+R_{\vartheta} \frac{\partial U_{4}}{\partial l_{4}}\left(R_{\vartheta} \vartheta^{a}\right)+R_{\vartheta} \frac{\partial U_{3}}{\partial l_{3}}\left(R_{\vartheta} \vartheta^{a}+R_{q} q\right)=\tau_{\vartheta}{ }^{a}
\end{array}\right.
$$

where $\tau$ is the torque applied at the joint side (for which no direct control is available and therefore to be interpreted as an external disturbing torque) and $\tau_{\vartheta}$ and $\tau_{\vartheta}^{a}$ represent the torques applied at the agonist and antagonist sides of the actuator (to be interpreted as internal actuation torques). As usual, the terms $U_{3}$ and $U_{4}$ represent the potential energy of two additional springs:

$$
\begin{array}{r}
U_{3}=U_{3}\left(l_{3}\right)=U_{3}\left(R_{q} q+R_{\vartheta} \vartheta^{a}\right) \\
U_{4}=U_{4}\left(l_{4}\right)=U_{4}\left(R_{\vartheta} \vartheta^{a}\right) .
\end{array}
$$

Assuming that constant torques are applied to the system $\left(\tau=\bar{\tau}, \tau_{\vartheta}=\bar{\tau}_{\vartheta}\right.$ and $\left.\tau_{\vartheta}^{a}=\bar{\tau}_{\vartheta}^{a}\right)$, equilibrium configurations $\left(q_{e q}, \vartheta_{e q}, \vartheta_{e q}^{a}\right)$ for the system should satisfy the following equation:

$$
\left\{\begin{array}{l}
-U_{1}^{\prime}(\hat{\vartheta})-U_{2}^{\prime}(\hat{\vartheta}+\hat{q})=\hat{\tau}_{\vartheta} \\
-U_{2}^{\prime}(\hat{\vartheta}+\hat{q})+U_{3}^{\prime}\left(-\hat{q}+\hat{\vartheta}^{a}\right)=\hat{\tau}_{q} \\
U_{4}^{\prime}\left(\hat{\vartheta}^{a}\right)+U_{3}^{\prime}\left(-\hat{q}+\hat{\vartheta}^{a}\right)=\hat{\tau}_{\vartheta}^{a}
\end{array}\right.
$$

where we defined:

$$
\begin{aligned}
& -R_{\vartheta} \vartheta_{e q}=\hat{\vartheta},-R_{q} q_{e q}=\hat{q}, R_{\vartheta} \vartheta_{e q}{ }^{a}=\hat{\vartheta}^{a}, \\
& \frac{\bar{\tau}_{\vartheta}}{R_{\vartheta}}=\hat{\tau}_{\vartheta}, \frac{\bar{\tau}_{q}}{R_{q}}=\hat{\tau}_{q}, \frac{\bar{\tau}_{\vartheta}^{a}}{R_{\vartheta}}=\hat{\tau}_{\vartheta}^{a} .
\end{aligned}
$$

Explicit calculation of the equilibrium configuration as a function of the input torques, i.e. explicit calculation of the functions $\hat{\vartheta}\left(\hat{\tau}_{\vartheta}, \hat{\tau}_{q}, \hat{\tau}_{\vartheta}^{a}\right), \hat{q}\left(\hat{\tau}_{\vartheta}, \hat{\tau}_{q}, \hat{\tau}_{\vartheta}^{a}\right)$, and $\hat{\vartheta}^{a}\left(\hat{\tau}_{\vartheta}, \hat{\tau}_{q}, \hat{\tau}_{\vartheta}^{a}\right)$ requires solving (3) which is a system of nonlinear equations (in three constraints and three unknowns). Its analytical solution might be quite challenging and therefore numerical solution results often necessary. Other quantities, such as the sensitivity of the equilibrium configuration to input torques, can be computed analytically by means of the implicit function theorem. Let's define:

$$
\alpha=\left[\begin{array}{c}
\hat{\vartheta} \\
\hat{\vartheta}^{a} \\
\hat{q}
\end{array}\right], \tau=\left[\begin{array}{c}
\hat{\tau}_{\vartheta} \\
\hat{\tau}_{\vartheta}^{a} \\
\hat{\tau}_{q}
\end{array}\right]
$$

and let's represent (3) in a compact way, with the following definition:

$$
\left\{\begin{array}{l}
-U_{1}^{\prime}(\hat{\vartheta})-U_{2}^{\prime}(\hat{\vartheta}+\hat{q})=\hat{\tau}_{\vartheta} \\
-U_{2}^{\prime}(\hat{\vartheta}+\hat{q})+U_{3}^{\prime}\left(\hat{\vartheta}^{a}-\hat{q}\right)=\hat{\tau}_{q} \quad \Longleftrightarrow f(\alpha, \tau)=0 . \\
U_{4}^{\prime}\left(\hat{\vartheta}^{a}\right)+U_{3}^{\prime}\left(\hat{\vartheta}^{a}-\hat{q}\right)=\hat{\tau}_{\vartheta}^{a}
\end{array}\right.
$$

By resourcing to the implicit function theorem, the equation $f(\alpha, \tau)=0$ locally defines a function $\alpha(\tau)$ (equilibrium configuration) with sensitivity:

$$
\frac{\partial \alpha}{\partial \tau}=-\left[\frac{\partial f}{\partial \alpha}\right]^{-1} \frac{\partial f}{\partial \tau}
$$

as easily follows by numerical derivation of the constrain equation $f(\alpha(\tau), \tau)=0$ :

$$
\frac{\partial f}{\partial \alpha} \frac{\partial \alpha}{\partial \tau}+\frac{\partial f}{\partial \tau}=0 \rightarrow \frac{\partial \alpha}{\partial \tau}=-\left[\frac{\partial f}{\partial \alpha}\right]^{-1} \frac{\partial f}{\partial \tau}
$$

Using the analytical expression of $f$ given by (3), we obtain:

$$
\frac{\partial f}{\partial \alpha}=\left[\frac{\partial f}{\partial \hat{\vartheta}} \frac{\partial f}{\partial \hat{\vartheta}^{a}} \frac{\partial f}{\partial \hat{q}}\right]=\left[\begin{array}{ccc}
-U_{1}^{\prime \prime}-U_{2}^{\prime \prime} & 0 & -U_{2}^{\prime \prime} \\
0 & U_{4}^{\prime \prime}+U_{3}^{\prime \prime} & -U_{3}^{\prime \prime} \\
-U_{2}^{\prime \prime} & U_{3}^{\prime \prime} & -U_{2}^{\prime \prime}-U_{3}^{\prime \prime}
\end{array}\right]
$$

and:

$$
\frac{\partial f}{\partial \tau}=\left[\begin{array}{ccc}
-1 & 0 & 0 \\
0 & -1 & 0 \\
0 & 0 & -1
\end{array}\right]
$$

which eventually results in the following expression:

$$
\frac{\partial \alpha}{\partial \tau}=\left[\begin{array}{ccc}
-U_{1}^{\prime \prime}-U_{2}^{\prime \prime} & 0 & -U_{2}^{\prime \prime} \\
0 & U_{4}^{\prime \prime}+U_{3}^{\prime \prime} & -U_{3}^{\prime \prime} \\
-U_{2}^{\prime \prime} & U_{3}^{\prime \prime} & -U_{2}^{\prime \prime}-U_{3}^{\prime \prime}
\end{array}\right]^{-1}
$$




$$
\begin{gathered}
\frac{\partial \alpha}{\partial \tau}=\left[\begin{array}{ccc}
\frac{\partial \hat{\vartheta}}{\partial \hat{\tau}_{\vartheta}} & \frac{\partial \hat{\vartheta}}{\partial \hat{\tau}_{\vartheta}^{a}} & \frac{\partial \hat{\vartheta}}{\partial \hat{\tau}_{q}} \\
\frac{\partial \hat{\vartheta}^{a}}{\partial \hat{\tau}_{\vartheta}} & \frac{\partial \hat{\vartheta}^{a}}{\partial \hat{\tau}_{\vartheta}^{a}} & \frac{\partial \hat{\vartheta}^{a}}{\partial \hat{\tau}_{q}} \\
\frac{\partial \hat{q}}{\partial \hat{\tau}_{\vartheta}} & \frac{\partial \hat{q}}{\partial \hat{\tau}_{\vartheta}^{a}} & \frac{\partial \hat{q}}{\partial \hat{\tau}_{q}}
\end{array}\right]= \\
{\left[\begin{array}{ccc}
-\left(U_{2}^{\prime \prime} U_{3}^{\prime \prime}+U_{2}^{\prime \prime} U_{4}^{\prime \prime}+U_{3}^{\prime \prime} U_{4}^{\prime \prime}\right) & -U_{2}^{\prime \prime} U_{3}^{\prime \prime} & U_{2}^{\prime \prime}\left(U_{3}^{\prime \prime}+U_{4}^{\prime \prime}\right) \\
U_{2}^{\prime \prime} U_{3}^{\prime \prime} & U_{1}^{\prime \prime} U_{2}^{\prime \prime}+U_{1}^{\prime \prime} U_{3}^{\prime \prime}+U_{2}^{\prime \prime} U_{3}^{\prime \prime} & -U_{3}^{\prime \prime}\left(U_{1}^{\prime \prime}+U_{2}^{\prime \prime}\right) \\
U_{2}^{\prime \prime}\left(U_{3}^{\prime \prime}+U_{4}^{\prime \prime}\right) & U_{3}^{\prime \prime}\left(U_{1}^{\prime \prime}+U_{2}^{\prime \prime}\right) & -\left(U_{1}^{\prime \prime}+U_{2}^{\prime \prime}\right)\left(U_{3}^{\prime \prime}+U_{4}^{\prime \prime}\right)
\end{array}\right]} \\
\frac{1}{U_{1}^{\prime \prime} U_{2}^{\prime \prime} U_{3}^{\prime \prime}+U_{1}^{\prime \prime} U_{2}^{\prime \prime} U_{4}^{\prime \prime}+U_{1}^{\prime \prime} U_{3}^{\prime \prime} U_{4}^{\prime \prime}+U_{2}^{\prime \prime} U_{3}^{\prime \prime} U_{4}^{\prime \prime}}
\end{gathered}
$$

The analytical expression for $\partial \alpha / \partial \tau$ will play a crucial role in the following sections. In particular, it will be used to design a control policy that maintains the joint equilibrium configuration (Section 4), to prove that this control policy monotonically increases the joint stiffness (Section 5), to compute the sensitivity of the equilibrium $\hat{q}$ with respect to the internal torques $\hat{\tau}_{\vartheta}$ and $\hat{\tau}_{\vartheta}^{a}$ (Section 6 ) and finally to synthesize a control policy for controlling the joint equilibrium configuration (Section 7).

\section{JOINT EQUILIBRIUM MAINTENANCE}

In this section we compute a control action which does not change the joint equilibrium configuration $\hat{q}$. This control action causes variation of the internal equilibrium configurations $\hat{\vartheta}$ and $\hat{\vartheta}^{a}$, which will eventually correspond to variations of the joint level stiffness (Section 5) and to variations of the sensitivity of $\hat{q}$ with respect to the internal toques, $\hat{\tau}_{\vartheta}$ and $\hat{\tau}_{\vartheta}^{a}$ (Section 6). Let's compute the time derivative of $\hat{q}$ as follows:

$$
\frac{d \hat{q}}{d t}=\frac{\partial \hat{q}}{\partial \hat{\tau}_{\vartheta}} \frac{d \hat{\tau_{\vartheta}}}{d t}+\frac{\partial \hat{q}}{\partial \hat{\tau}_{\vartheta}^{a}} \frac{d \hat{\tau}_{\vartheta}^{a}}{d t}+\frac{\partial \hat{q}}{\partial \hat{\tau}_{q}} \frac{d \hat{\tau}_{q}}{d t} .
$$

Given that we do not have direct control on $\hat{\tau}_{q}$, let's assume that $d \hat{\tau}_{q} / d t=0$ so that the time derivative of $\hat{q}$ results to be:

$$
\frac{d \hat{q}}{d t}=\underbrace{\left[\begin{array}{ccc}
\frac{\partial \hat{q}}{\partial \hat{\tau}_{\vartheta}} & \frac{\partial \hat{q}}{\partial \hat{\tau}_{\vartheta}^{a}} & \frac{\partial \hat{q}}{\partial \hat{\tau}_{q}}
\end{array}\right]}_{N} \underbrace{\left[\begin{array}{c}
\frac{d \hat{\tau}_{\vartheta}}{d t} \\
\frac{d \tau_{\vartheta}^{a}}{d t} \\
0
\end{array}\right]}_{\delta \tau},
$$

where $\delta \tau$ is the time derivative of the old control variable $\tau$, i.e. $\delta \tau=d \tau / d t$. In order to have constant value for $\hat{q}$, we should satisfy the equation $N \cdot \delta \tau=0$ where an analytical expression for $N$ is given by the last row of the matrix $\partial \alpha / \partial \tau$. Substitutions show that equation (5) is satisfied by the following space of controls:

$$
\delta \tau=\left[\begin{array}{c}
-U_{3}^{\prime \prime}\left(U_{1}^{\prime \prime}+U_{2}^{\prime \prime}\right) \\
U_{2}^{\prime \prime}\left(U_{3}^{\prime \prime}+U_{4}^{\prime \prime}\right) \\
0
\end{array}\right] \cdot u \cdot k, \quad \forall u \in \mathbb{R}
$$

with:

$$
k=U_{1}^{\prime \prime} U_{2}^{\prime \prime} U_{3}^{\prime \prime}+U_{1}^{\prime \prime} U_{2}^{\prime \prime} U_{4}^{\prime \prime}+U_{1}^{\prime \prime} U_{3}^{\prime \prime} U_{4}^{\prime \prime}+U_{2}^{\prime \prime} U_{3}^{\prime \prime} U_{4}^{\prime \prime} .
$$

For such control actions, the resulting time evolution of the system equilibrium $\alpha$ is given by the following expression:

$$
\frac{d \alpha}{d t}=\frac{\partial \alpha}{\partial \tau} \delta \tau=\left[\begin{array}{c}
U_{3}^{\prime \prime}\left(\hat{\vartheta}^{a}-\hat{q}\right) \\
U_{2}^{\prime \prime}(\hat{\vartheta}-\hat{q}) \\
0
\end{array}\right] \cdot u
$$

where we explicitly indicated the dependence of $U_{2}(\cdot)$ and $U_{3}(\cdot)$ on $\hat{\vartheta}^{a}$, $\hat{q}$ and $\hat{\vartheta}$.

\subsection{Joint position maintenance}

Changing the stiffness with the control strategy (6) has the drawback of moving $q$ even if $d \hat{q} / d t=0$, since the system traverses states which are not at equilibrium. Another strategy, proposed in this section, consists in selecting torques at the motors such that $\ddot{q}=0$. Starting from the dynamic equation of $q$ :

$$
I_{q} \ddot{q}-R_{q} U_{2}^{\prime}\left(-R_{\vartheta} \vartheta-R_{q} q\right)+R_{q} U_{3}^{\prime}\left(R_{q} q+R_{\vartheta} \vartheta^{a}\right)=\tau_{q},
$$

we need to impose:

$$
-U_{2}^{\prime}\left(-R_{\vartheta} \vartheta-R_{q} q\right)+U_{3}^{\prime}\left(R_{q} q+R_{\vartheta} \vartheta^{a}\right)=0
$$

which will be sufficient to guarantee that $\ddot{q}=0$, if the following initial conditions are satisfied: $\dot{q}(0)=0, q(0)=\bar{q}$. Doing the usual change of variables:

$$
\begin{aligned}
& -R_{\vartheta} \vartheta_{e q}=\bar{\vartheta},-R_{q} q_{e q}=\bar{q}, R_{\vartheta} \vartheta_{e q}{ }^{a}=\bar{\vartheta}^{a}, \\
& \frac{\bar{\tau}_{\vartheta}}{R_{\vartheta}}=\bar{\tau}_{\vartheta}, \frac{\bar{\tau}_{q}}{R_{q}}=\bar{\tau}_{q}, \frac{\bar{\tau}_{\vartheta}^{a}}{R_{\vartheta}}=\bar{\tau}_{\vartheta}^{a} .
\end{aligned}
$$

we have:

$$
-U_{2}^{\prime}(\bar{\vartheta}+\bar{q})+U_{3}^{\prime}\left(\bar{\vartheta}^{a}-\bar{q}\right)=0
$$

which implicitly defines a local relationship $\bar{\vartheta}^{a}(\bar{\vartheta})$ (once again thanks to the implicit function theorem). Deriving twice the expression above we obtain:

$$
\frac{d^{2} \bar{\vartheta}^{a}}{d t}=\frac{d}{d t}\left(\frac{U_{2}^{\prime \prime}}{U_{3}^{\prime \prime}}\right) \frac{d \bar{\vartheta}}{d t}+\frac{U_{2}^{\prime \prime}}{U_{3}^{\prime \prime}}\left(\frac{d^{2} \bar{\vartheta}}{d t^{2}}\right)
$$

where the accelerations of $\bar{\vartheta}^{a}$ and $\bar{\vartheta}$ can be retrieved from the following equations:

$$
\begin{aligned}
& \bar{\tau}_{\vartheta}=-I_{\vartheta} \ddot{\bar{\vartheta}}-U_{1}^{\prime}(\bar{\vartheta})-U_{2}^{\prime}(\bar{\vartheta}+\bar{q}), \\
& \bar{\tau}_{\vartheta}^{a}=I_{\vartheta} \ddot{\bar{\vartheta}}^{a}+U_{4}^{\prime}\left(\bar{\vartheta}^{a}\right)+U_{3}^{\prime}\left(\bar{\vartheta}^{a}-\bar{q}\right) .
\end{aligned}
$$

Easy substitutions lead to:

$$
\begin{aligned}
\bar{\tau}_{\vartheta}^{a} & =I_{\vartheta}\left(\frac{d}{d t}\left(\frac{U_{2}^{\prime \prime}}{U_{3}^{\prime \prime}}\right) \frac{d \bar{\vartheta}}{d t}+\frac{U_{2}^{\prime \prime}}{U_{3}^{\prime \prime}}\left(\frac{d^{2} \bar{\vartheta}}{d t^{2}}\right)\right)+U_{4}^{\prime}+U_{3}^{\prime} \\
& =I_{\vartheta}\left(\frac{d}{d t}\left(\frac{U_{2}^{\prime \prime}}{U_{3}^{\prime \prime}}\right) \frac{d \bar{\vartheta}}{d t}\right)+U_{4}^{\prime}+U_{3}^{\prime}-\frac{U_{2}^{\prime \prime}}{U_{3}^{\prime \prime}}\left(\bar{\tau}_{\vartheta}+U_{1}^{\prime}+U_{2}^{\prime}\right)
\end{aligned}
$$

This last expression in practice gives the torque $\tau_{\vartheta}^{a}$ to give to the antagonist muscle as a function of the torque given to the agonist, $\tau_{\vartheta}$, in order to maintain the position of $q$ unchanged. If this control strategy is applied in between two equilibrium configurations (null velocities and accelerations), it is easy to conclude the equilibrium of $q$ should not be changed and therefore the control strategy that we just proposed can be also used, equivalently to (6), to move the system across equilibrium states that do not change $\hat{q}$. 


\section{JOINT LEVEL STIFFNESS}

In this section we compute the joint level stiffness, defined as the sensitivity of the equilibrium configuration $\hat{q}$ with respect to variations of the external torque $\hat{\tau} q$. Analytically, this quantity coincides with $\partial \hat{q} / \partial \hat{\tau}_{q}$ and therefore it can be extracted as the element $(3,3)$ in the matrix $\partial \alpha / \partial \tau$ :

$$
\frac{\partial \hat{q}}{\partial \hat{\tau}_{q}}=-\frac{U_{1}^{\prime \prime} U_{2}^{\prime \prime} U_{3}^{\prime \prime}+U_{1}^{\prime \prime} U_{2}^{\prime \prime} U_{4}^{\prime \prime}+U_{3}^{\prime \prime} U_{4}^{\prime \prime} U_{1}^{\prime \prime}+U_{1}^{\prime \prime} U_{3}^{\prime \prime} U_{4}^{\prime \prime}}{\left(U_{1}^{\prime \prime}+U_{2}^{\prime \prime}\right)\left(U_{3}^{\prime \prime}+U_{4}^{\prime \prime}\right)} .
$$

Easy calculations show that we have:

$$
\frac{\partial \hat{q}}{\partial \hat{\tau}_{q}}=-\frac{1}{\frac{1}{\frac{1}{U_{1}^{\prime \prime}}+\frac{1}{U_{2}^{\prime \prime}}}+\frac{1}{\frac{1}{U_{3}^{\prime \prime}}+\frac{1}{U_{4}^{\prime \prime}}}}
$$

This last equation represents the intuitive result that the joint stiffness is the series of parallel of springs, nominally the series of $U_{1}, U_{2}$ in parallel with the series of $U_{3}, U_{4}$ seen in Eq. (2). This property can be exploited to find a simple way to increase monotonically the system stiffness (which is desirable for disturbance rejection). It is indeed intuitive to conclude that global stiffness will increase if all individual stiffnesses $\left(U_{1}, U_{2}, U_{3}, U_{4}\right)$ are increased. A control strategy to increase all stiffnesses is hereafter described. Let's consider the control action Eq. (6) that maintains the equilibrium position $\hat{q}$. According to (8), this control action corresponds to the following internal variables displacement:

$$
\begin{gathered}
d \hat{\vartheta} / d t=U_{3}^{\prime \prime}\left(\hat{\vartheta}^{a}-\hat{q}\right) \\
d \hat{\vartheta}^{a} / d t=U_{2}^{\prime \prime}(\hat{\vartheta}+\hat{q}) .
\end{gathered}
$$

If $U_{2}^{\prime \prime}>0$ and $U_{3}^{\prime \prime}>0$ in the entire workspace, we can easily conclude that both $\hat{\vartheta}^{a}$ and $\hat{\vartheta}$ are increasing under the control action (6). Moreover, if $U_{1}^{\prime \prime \prime}>0, U_{2}^{\prime \prime \prime}>0, U_{3}^{\prime \prime \prime}>0$ and $U_{4}^{\prime \prime \prime}>0$, we can conclude that this control action is increasing individual stiffnesses thus corresponding to a monotonically increasing value for $\partial \hat{\tau}_{q} / \partial \hat{q}$, i.e. the joint level stiffness.

\section{SENSITIVITY OF EQUILIBRIUM TO INTERNAL ACTUATION TORQUES}

In this section we focus our attention on the quantity $\partial \hat{q} / \partial \hat{\tau}_{\vartheta}$ which represents the sensitivity of the joint position $\hat{q}$ with respect to the internal torque $\hat{\tau}_{\vartheta}$. Thanks to the symmetry of the system, the properties hereafter discussed will hold for the analogous quantity $\partial \hat{q} / \partial \hat{\tau}_{\vartheta}$. From the expression of $\partial \alpha / \partial \tau$ given in Section 3, we have:

$$
\frac{\partial \hat{q}}{\partial \hat{\tau}_{\vartheta}}=\frac{U_{2}^{\prime \prime}}{U_{1}^{\prime \prime}+U_{2}^{\prime \prime}} \frac{\partial \hat{q}}{\partial \hat{\tau}_{q}}
$$

and inverting the expression above we have:

$$
\left(\frac{\partial \hat{q}}{\partial \hat{\tau}_{\vartheta}}\right)^{-1}=\underbrace{\frac{U_{1}^{\prime \prime}+U_{2}^{\prime \prime}}{U_{2}^{\prime \prime}}}_{g(\hat{\vartheta}, \hat{q})} \underbrace{\left(\frac{\partial \hat{q}}{\partial \hat{\tau}_{q}}\right)^{-1}}_{\text {joint stiffness }} .
$$

In the following we give sufficient (but not necessary) conditions for guaranteeing that the control action (6) leads to decreasing values of either $\partial \hat{q} / \partial \hat{\tau}_{\vartheta}$ or $\partial \hat{q} / \partial \hat{\tau}_{\vartheta^{a}}$. At the control level, this is a desirable property because it allows to have finer control over the variable $\hat{q}$ (since identical variations in the control variables $\tau_{\vartheta}, \tau_{\vartheta}^{a}$ will correspond to smaller steps in the equilibrium configuration for $\hat{q}$ ). From (11), we notice that this property is guaranteed if both the joint stiffness and the function $g(\vartheta, q)$ are increasing. From Section 5 , we already know that both the joint stiffness and the variables $\hat{\vartheta}^{a}$ and $\hat{\vartheta}$ are increasing under the control action (6), which keeps $\hat{q}$ constant. Therefore we are left with guaranteeing that $g(\hat{\vartheta}, \hat{q})$ is non-decreasing in $\hat{\vartheta}$. Easy computations show that:

$$
\frac{\partial g}{\partial \hat{\vartheta}}>0 \quad \Longleftrightarrow \quad \frac{U_{1}^{\prime \prime}}{U_{1}^{\prime \prime \prime}}<\frac{U_{2}^{\prime \prime}}{U_{2}^{\prime \prime \prime}} .
$$

In case $U_{1}=U_{2}=U$, the above condition can be written as follows:

$$
\frac{U^{\prime \prime}(\hat{\vartheta})}{U^{\prime \prime \prime}(\hat{\vartheta})}<\frac{U^{\prime \prime}(\hat{\vartheta}+\hat{q})}{U^{\prime \prime \prime}(\hat{\vartheta}+\hat{q})}
$$

If $\hat{q}>0$, we are left with guaranteeing that the function $U^{\prime \prime} / U^{\prime \prime \prime}$ is monotonically increasing. If $\hat{q}<0$, we can consider $\partial \hat{q} / \partial \hat{\tau}_{\vartheta^{a}}$ and a similar property will hold in consequence of the fact that $U_{3}$ is a function of $\hat{\vartheta}^{a}-\hat{q}$. In practice, what we have just given are conditions for guaranteeing that the control action (6) leads to finer control of $q$ via $\tau_{\vartheta}^{a}$ or $\tau_{\vartheta}$, the choice depending on which of the two actuators (agonist $U_{1}, U_{2}$ or antagonist $U_{3}, U_{4}$ ) is pulling more.

\section{POSITION CONTROLLER}

In this section we describe how to control the equilibrium configuration of the joint variable $q$. Starting from (5), we can try to find solutions to the following equation $d \hat{q} / d t=$ $N \delta \tau=v$ so as to define a new control input $v$ which directly acts on time variations of $\hat{q}$. The generic solution of this linear problem (imposing, as usual, direct control only on the internal torques) is given by the following (which is an extension of $(6))$ :

$\delta \tau=\left[\begin{array}{c}U_{2}^{\prime \prime}\left(U_{3}^{\prime \prime}+U_{4}^{\prime \prime}\right) \\ U_{3}^{\prime \prime}\left(U_{1}^{\prime \prime}+U_{2}^{\prime \prime}\right) \\ 0\end{array}\right] \cdot v \cdot k_{1}+\left[\begin{array}{c}-U_{3}^{\prime \prime}\left(U_{1}^{\prime \prime}+U_{2}^{\prime \prime}\right) \\ U_{2}^{\prime \prime}\left(U_{3}^{\prime \prime}+U_{4}^{\prime \prime}\right) \\ 0\end{array}\right] \cdot u \cdot k_{2}$,

with:

$$
\begin{aligned}
& k_{2}=U_{1}^{\prime \prime} U_{2}^{\prime \prime} U_{3}^{\prime \prime}+U_{1}^{\prime \prime} U_{2}^{\prime \prime} U_{4}^{\prime \prime}+U_{1}^{\prime \prime} U_{3}^{\prime \prime} U_{4}^{\prime \prime}+U_{2}^{\prime \prime} U_{3}^{\prime \prime} U_{4}^{\prime \prime}, \\
& k_{1}=k_{2}^{2}\left(\left(U_{2}^{\prime \prime}\left(U_{3}^{\prime \prime}+U_{4}^{\prime \prime}\right)\right)^{2}+\left(U_{3}^{\prime \prime}\left(U_{1}^{\prime \prime}+U_{2}^{\prime \prime}\right)\right)^{2}\right) .
\end{aligned}
$$

The new control variable $v$ acts directly on the time derivative of $\hat{q}$, while $u$ monotonically changes the joint stiffness maintaining a constant value for $\hat{q}$.

\section{SIMULATIONS}

We tested the proposed analysis on a simulation of the actuator represented in Fig. 3 with additional damping for making the system stable (please contact the authors for a complete list of the parameters used in the simulation). Selected springs are cubic $U_{1}(x)=U_{2}(x)=U_{3}(x)=$ $U_{4}(x)=1 / 3 x^{3}$ and therefore satisfy all the required conditions outlined in Section 5 and 6 when used in 

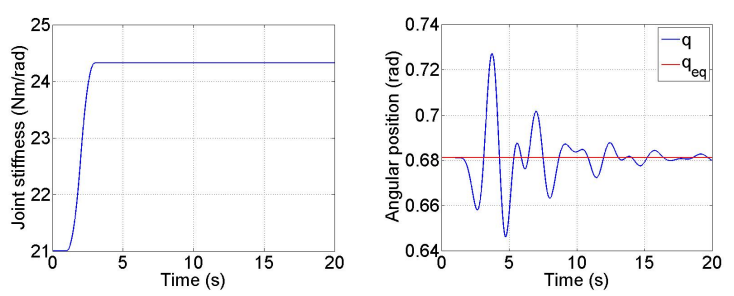

Fig. 4. Left graph: joint stiffness variation. Right graph: joint position $q$ (in blue) and joint equilibrium $\hat{q}$ (in red) variations in response to the control action (6).

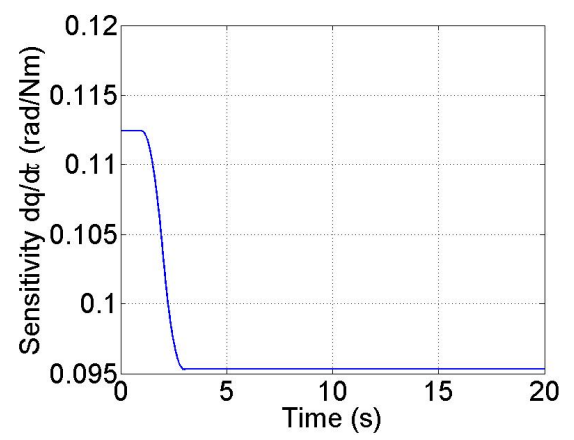

Fig. 5. Variation of the sensitivity $\partial \hat{q} / \partial \hat{\tau}_{\vartheta^{a}}$ in response to the control action (6).

elongation $(x>0)$. Responses to a control action (6) are as expected, in response to an input $u$ described as follows: $u(t)=t-1, t \in[1,2], u(t)=-t+3, t \in[2,3]$ and $u(t)=0$ otherwise. The left graph in Fig. 4 plots the joint stiffness, while the right graph shows the joint equilibrium configuration $\hat{q}$ (in red, constant as expected), and the corresponding joint position $q$ transient response (in blue). The sensitivity $\partial \hat{q} / \partial \hat{\tau}_{\vartheta^{a}}$ is instead plotted in Fig. 5 , and as expected the variation in response to the control action (6) leads to a monotonically decreasing value for the sensitivity of $\hat{q}$ with respect to $\hat{\tau}_{\vartheta a}$.

\section{CONCLUSION}

In this paper we characterized some important control properties of a novel variable stiffness actuator. The proposed design takes inspiration from the agonist-antagonist configuration of muscles in biological systems. The design is based on custom non-linear springs, whose potential energy was left unspecified in the theoretical analysis. After a characterization of the system stiffness, we proposed a control action (6) sufficient for guaranteeing a monotonically increasing joint stiffness, a desirable property for augmenting the system disturbance rejection. This property was guaranteed with minimal requirements on the spring potential energy (basically positiveness of the derivatives). Another analyzed property was the sensitivity of joint equilibrium position with respect to the control inputs; again, sufficient conditions on the spring potential energy were given in order to guarantee that the control action (6) was monotonically decreasing this sensitivity (a desirable property when trying to control joint position at a finer scale). A realization of the proposed actuator is foreseen to test the utility of these theoretical results on the real system.

\section{ACKNOWLEDGEMENTS}

This work was supported in part by the European Projects: VIACTORS (FP7-ICT-2007-3), CHRIS (FP7IST-215805) and ITALK (ICT-214668).

\section{REFERENCES}

Berret, B., Ivaldi, S., Nori, F., and Sandini, G. (2011). Stochastic optimal control with variable impedance manipulators in presence of uncertainties and delayed feedback. In Intelligent Robots and Systems (IROS), 2011 IEEE/RSJ International Conference on, 43544359. IEEE.

Burdet, E., Osu, R., Franklin, D.W., Milner, T.E., and Kawato, M. (2001). The central nervous system stabilizes unstable dynamics by learning optimal impedance. Nature, 414(6862), 446-9. doi:10.1038/35106566.

Feldman, A. (1966). Functional tuning of the nervous system with control of movement or maintenance of a steady posture. Biophysics, 565-578.

Franklin, D.W., Liaw, G., Milner, T.E., Osu, R., Burdet, E., and Kawato, M. (2007). Endpoint stiffness of the arm is directionally tuned to instability in the environment. Journal of Neuroscience, 27(29), 7705-7716.

Ham, R., Sugar, T., Vanderborght, B., Hollander, K., and Lefeber, D. (2009). Compliant actuator designs. IEEE Robotics Automation Magazine, 16(3), 81-94.

Hill, A. and Gasser, H. (1924). The dynamics of muscular contraction. Proc $R$ Soc London, 398-437.

Jafari, A., Tsagarakis, N.G., Vanderborght, B., and Caldwell, D.G. (2010). A Novel Actuator with Adjustable Stiffness ( AwAS ). Technology, 4201-4206. doi: 10.1109/IROS.2010.5648902.

McMahon, T. (1984). Muscle, Reflexes, and Locomotion. 